\title{
An extension of Saalschütz's summation theorem for the series ${ }_{r+3} F_{r+2}$
}

\author{
Y. S. KIM ${ }^{a}$, A. K. RAthiE ${ }^{b}$ AND R. B. PARIS ${ }^{c}$ \\ ${ }^{a}$ Department of Mathematics Education, Wonkwang University, Iksan, Korea \\ E-Mail: yspkim@wonkwang.ac.kr \\ ${ }^{b}$ School of Mathematical and Physical Sciences, Central University of Kerala, \\ Kasaragad 671328, Kerala, India \\ E-Mail: akrathie@rediffmail.com \\ ${ }^{c}$ University of Abertay Dundee, Dundee DD1 1HG, UK \\ E-Mail: r.paris@abertay.ac.uk
}

\begin{abstract}
The aim in this research note is to provide an extension of Saalschütz's summation theorem for the series ${ }_{r+3} F_{r+2}(1)$ when $r$ pairs of numeratorial and denominatorial parameters differ by positive integers. The result is obtained by exploiting a generalization of an Eulertype transformation recently derived by Miller and Paris [9].
\end{abstract}

Mathematics Subject Classification: 33C15, 33C20

Keywords: Generalized hypergeometric series, unit argument, Saalschütz's theorem

\section{Introduction}

The generalized hypergeometric function ${ }_{p} F_{q}(x)$ may be defined for complex parameters and argument by the series

$$
{ }_{p} F_{q}\left(\begin{array}{c}
a_{1}, a_{2}, \ldots, a_{p} \\
b_{1}, b_{2}, \ldots, b_{q}
\end{array} \mid x\right)=\sum_{k=0}^{\infty} \frac{\left(a_{1}\right)_{k}\left(a_{2}\right)_{k} \ldots\left(a_{p}\right)_{k}}{\left(b_{1}\right)_{k}\left(b_{2}\right)_{k} \ldots\left(b_{q}\right)_{k}} \frac{x^{k}}{k !} .
$$

When $q=p$ this series converges for $|x|<\infty$, but when $q=p-1$ convergence occurs when $|x|<1$. However, when only one of the numeratorial parameters $a_{j}$ is a negative integer or zero, then the series always converges since it is simply a polynomial in $x$ of degree $-a_{j}$. In (1.1) the Pochhammer symbol or ascending factorial $(a)_{n}$ is given by

$$
(a)_{n}=\frac{\Gamma(a+n)}{\Gamma(a)}= \begin{cases}1 & (n=0) \\ a(a+1) \ldots(a+n-1) & (n \in \mathbf{N}),\end{cases}
$$

where $\Gamma$ is the gamma function. The parametric excess $s$ of the above series is defined by

$$
s=\sum_{r=1}^{q} b_{r}-\sum_{r=1}^{p} a_{r}
$$


We shall adopt the convention of writing the finite sequence of parameters $\left(a_{1}, \ldots, a_{p}\right)$ simply by $\left(a_{p}\right)$ and the product of $p$ Pochhammer symbols by

$$
\left(\left(a_{p}\right)\right)_{k} \equiv\left(a_{1}\right)_{k} \ldots\left(a_{p}\right)_{k}
$$

where an empty product $p=0$ reduces to unity. It is evident that whenever generalized hypergeometric functions of special argument reduce to Gamma functions the results are of considerable importance in applications. Until 1990, only a few classical summation theorems for ${ }_{2} F_{1},{ }_{3} F_{2}$ and for higher order series were known. Subsequently, some progress has been made in generalizing these classical summation theorems; see $[1,3,4,5,7,10,13,15]$.

In our present investigation we shall be concerned with the following summation theorem due to Saalschütz [14, p. 49]

$$
{ }_{3} F_{2}\left(\begin{array}{c|c}
-n, a, b \\
c, 1+a+b-c-n
\end{array} \mid 1\right)=\frac{(c-a)_{n}(c-b)_{n}}{(c)_{n}(c-a-b)_{n}},
$$

where $n$ is a nonnegative integer. Such a series is said to be Saalschützian since the parametric excess $s=1$. As shown in [14, p. 49], this follows from taking the well-known Euler transformation

$$
(1-x)^{a+b-c}{ }_{2} F_{1}\left(\begin{array}{c|c}
a, b & x \\
c & x
\end{array}\right)={ }_{2} F_{1}\left(\begin{array}{c|c}
c-a, c-b & x \\
c & \mid
\end{array}\right)
$$

and equating coefficients of $x^{n}$ on both sides of the equation. An extension of Saalschütz's theorem has been considered recently by Rakha and Rathie in [13] who showed that

$$
{ }_{4} F_{3}\left(\begin{array}{c|c}
-n, a, b, & f+1 \\
c, 2+a+b-c-n, & f
\end{array}\right)=\frac{(c-a-1)_{n}(c-b-1)_{n}}{(c)_{n}(c-a-b-1)_{n}} \frac{(\eta+1)_{n}}{(\eta)_{n}},
$$

where

$$
\eta=\frac{(c-a-1)(c-b-1) f}{a b+(c-a-b-1) f} .
$$

As an application of this result these authors also established the quadratic transformation

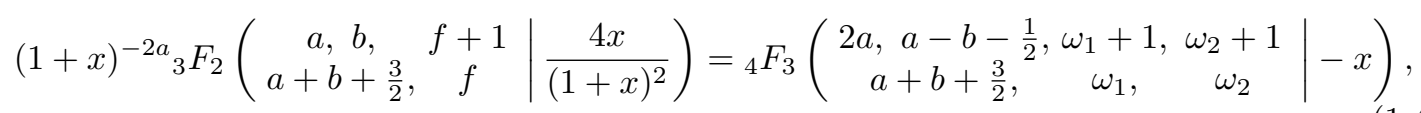

where

$$
\omega_{1,2}=a \pm\left[a^{2}+\left(a+\frac{1}{2}\right)\left(a+b-\frac{1}{2}\right) f /(b-f)\right]^{1 / 2}
$$

In [9], a generalization of the Euler transformation (1.3), when $r$ pairs of numeratorial and denominatorial parameters differ by positive integers $\left(m_{r}\right)$, was obtained in the form

$$
\begin{aligned}
{ }_{r+2} F_{r+1}\left(\begin{array}{cc}
a, b, & \left(f_{r}+m_{r}\right) \\
c, & \left(f_{r}\right)
\end{array} \mid x\right) \\
\quad=(1-x)^{c-a-b-m}{ }_{m+2} F_{m+1}\left(\begin{array}{c}
c-a-m, c-b-m, \\
c, \\
\left(\eta_{m}+1\right) \\
\left(\eta_{m}\right)
\end{array} \mid x\right)
\end{aligned}
$$

when $|x|<1$. Here $\left(\eta_{m}\right)$ are the nonvanishing zeros of the associated parametric polynomial $Q_{m}(t)$ of degree $m \equiv m_{1}+\cdots+m_{r}$ given by

$$
Q_{m}(t)=\sum_{k=0}^{m} B_{k}(a)_{k}(b)_{k}(t)_{k} G_{m, k}(t)
$$


with

$$
B_{k} \equiv(-1)^{k} A_{k}(c-a-m+k)_{m-k}(c-b-m+k)_{m-k}
$$

and

$$
G_{m, k}(t) \equiv{ }_{3} F_{2}\left(\begin{array}{c}
-m+k, t+k, c-a-b-m \\
c-a-m+k, c-b-m+k
\end{array} \mid 1\right) .
$$

For $0 \leq k \leq m$, the function $G_{m, k}(t)$ is a polynomial in $t$ of degree $m-k$. The coefficients $A_{k}$ are defined by

$$
A_{k}=\sum_{j=k}^{m} \mathbf{S}_{j}^{(k)} \sigma_{m-j}, \quad A_{0}=\left(f_{1}\right)_{m_{1}} \ldots\left(f_{r}\right)_{m_{r}}, \quad A_{m}=1,
$$

where $\mathbf{S}_{j}^{(k)}$ is the Stirling number of the second kind and the coefficients $\sigma_{j}(0 \leq j \leq m)$ are generated by

$$
\left(f_{1}+x\right)_{m_{1}} \ldots\left(f_{r}+x\right)_{m_{r}}=\sum_{j=0}^{m} \sigma_{m-j} x^{j} .
$$

The case $m_{1}=\cdots=m_{r}=1$ in (1.5) has been given earlier in [8], and also in [6] using different methods. When $\left(m_{r}\right)$ is empty we define $m=0$; in this case (1.5) reduces to the Euler transformation (1.3). In Section 2, we shall employ the same approach described in [14, p. 49] to the transformation formula (1.5) in our proof of the extension of Saalschütz's theorem to ${ }_{r+3} F_{r+2}$ series.

\section{The extension of Saalschütz's theorem}

The extension of Saalschütz's summation theorem is given by the following:

Theorem 1. Let $\left(m_{r}\right)$ be a set of positive integers and define $m \equiv m_{1}+\cdots+m_{r}$. Let $n$ be a nonnegative integer. Then

$$
{ }_{r+3} F_{r+2}\left(\begin{array}{c}
-n, a, b, \\
c, 1+a+b-c+m-n, \quad\left(f_{r}+m_{r}\right)
\end{array} \mid 1\right)=\frac{(c-a-m)_{n}(c-b-m)_{n}}{\left(f_{r}\right)} \frac{\left(\left(\eta_{m}+1\right)\right)_{n}}{\left(\left(\eta_{m}\right)\right)_{n}(c-a-b-m)_{n}},
$$

where $\left(\eta_{m}\right)$ are the nonvanishing zeros of the associated parametric polynomial $Q_{m}(t)$ of degree $m$ defined in (1.6).

Proof: From (1.5) we have

$$
\begin{aligned}
{ }_{m+2} F_{m+1}\left(\begin{array}{rl}
c-a-m, c-b & -m, \\
c, & \left(\eta_{m}+1\right) \\
\left(\eta_{m}\right)
\end{array} \mid x\right) \\
\quad=(1-x)^{m+a+b-c}{ }_{r+2} F_{r+1}\left(\begin{array}{cc}
a, b, & \left(f_{r}+m_{r}\right) \\
c, & \left(f_{r}\right)
\end{array} \mid x\right) .
\end{aligned}
$$

The coefficient of $x^{n}$ on the left-hand side is

$$
\frac{(c-a-m)_{n}(c-b-m)_{n}}{(c)_{n} n !} \frac{\left(\left(\eta_{m}+1\right)\right)_{n}}{\left(\left(\eta_{m}\right)\right)_{n}}
$$

and this must equal the coefficient of $x^{n}$ on the right-hand side. With

$$
D_{k} \equiv(-1)^{k}\left(\begin{array}{c}
m+a+b-c \\
k
\end{array}\right),
$$


this latter coefficient is given by

$$
\begin{aligned}
\sum_{k=0}^{n} D_{n-k} & \frac{(a)_{k}(b)_{k}}{(c)_{k} k !} \frac{\left(\left(f_{r}+m_{r}\right)\right)_{k}}{\left(\left(f_{r}\right)\right)_{k}} \\
& =\sum_{k=0}^{n} \frac{(-1)^{n-k} \Gamma(1+a+b-c+m)}{(n-k) ! \Gamma(1+a+b-c+m-n+k)} \frac{(a)_{k}(b)_{k}}{(c)_{k} k !} \frac{\left(\left(f_{r}+m_{r}\right)\right)_{k}}{\left(\left(f_{r}\right)\right)_{k}} \\
& =\frac{(c-a-b-m)_{n}}{n !} \sum_{k=0}^{n} \frac{(-n)_{k}}{k !} \frac{(a)_{k}(b)_{k}}{(c)_{k}(1+a+b-c+m-n)_{k}} \frac{\left(\left(f_{r}+m_{r}\right)\right)_{k}}{\left(\left(f_{r}\right)\right)_{k}} \\
& =\frac{(c-a-b-m)_{n}}{n !}{ }_{r+3} F_{r+2}\left(\begin{array}{c}
c, 1+a+b-c+b, \quad-n-n, \quad\left(f_{r}+m_{r}\right) \\
\left(f_{r}\right)
\end{array}\right),
\end{aligned}
$$

where we have used the identities

$$
(-n)_{k}=\frac{(-1)^{k} n !}{(n-k) !}, \quad \frac{\Gamma(1-\alpha)}{\Gamma(1-\alpha-n)}=(-1)^{n}(\alpha)_{n}
$$

Equating this coefficient to that in (2.2) we then obtain the desired summation (2.1)

\section{Examples}

In the case $r=1$ and $m_{1}=m=1$ we have from $(2.1)$

$$
{ }_{4} F_{3}\left(\begin{array}{c|c}
-n, a, b, & f+1 \\
c, 2+a+b-c-n, & f
\end{array}\right)=\frac{(c-a-1)_{n}(c-b-1)_{n}}{(c)_{n}(c-a-b-1)_{n}} \frac{(\eta+1)_{n}}{(\eta)_{n}},
$$

where $\eta$ is the zero of the first-degree parametric polynomial obtained from (1.6)

$$
Q_{1}(t)=-\{f(c-a-b-1)+a b\} t+(c-a-1)(c-b-1) f,
$$

whence

$$
\eta=\frac{(c-a-1)(c-b-1) f}{a b+(c-a-b-1) f} .
$$

This result was derived in [13] using a different approach.

A special case of (3.1), which may be of some interest, is obtained when $c=1+a-b$, so that $\eta=(a-2 b) f /(2 f-a)$, to yield

$$
{ }_{4} F_{3}\left(\begin{array}{cc|c}
-n, a, b, & f+1 \\
1+a-b, 1+2 b-n, & f & 1
\end{array}\right)=\frac{(a-2 b)_{n}(-b)_{n}}{(1+a-b)_{n}(-2 b)_{n}}\left\{1+\frac{n(2 f-a)}{(a-2 b) f}\right\},
$$

where we have used the fact that $(\eta+1)_{n} /(\eta)_{n}=(\eta+n) / \eta$. This result was obtained earlier by Kim, Rathie and Paris by other means who used it to obtain a reduction formula for the Kampé de Fériet function [2]. In the above result, if we let $f=\frac{1}{2} a$ we obtain

$$
{ }_{4} F_{3}\left(\begin{array}{c|c}
-n, a, b, 1+\frac{1}{2} a \\
c, 1+2 b-n, \frac{1}{2} a
\end{array} \mid 1\right)=\frac{(a-2 b)_{n}(-b)_{n}}{(1+a-b)_{n}(-2 b)_{n}} \quad(a \neq 2 b),
$$

which is a known summation; see [14, Appendix III, Eq. (17)]. 
Also, in the case $r=1$ with $m=2$, we find from (1.7) that $A_{0}=f(1+f), A_{1}=2(1+f)$ and $A_{2}=1$. Introducing the abbreviated notation

$$
\lambda \equiv c-a-2, \quad \lambda^{\prime} \equiv c-b-2, \quad \sigma \equiv c-a-b-2,
$$

we obtain from (1.6) the quadratic parametric polynomial (with zeros $\eta_{1}$ and $\eta_{2}$ ) given by

$$
\begin{gathered}
Q_{2}(t)=A_{0}(\lambda)_{2}\left(\lambda^{\prime}\right)_{2}\left\{1-\frac{2 \sigma t}{\lambda \lambda^{\prime}}+\frac{(t)_{2}(\sigma)_{2}}{(\lambda)_{2}\left(\lambda^{\prime}\right)_{2}}\right\}-A_{1} a b t(\lambda+1)\left(\lambda^{\prime}+1\right)\left\{1-\frac{\sigma(1+t)}{(\lambda+1)\left(\lambda^{\prime}+1\right)}\right\} \\
+A_{2}(a)_{2}(b)_{2} t(1+t) \\
=A_{0}(\lambda)_{2}\left(\lambda^{\prime}\right)_{2}\left\{1-\frac{2 B t}{\lambda \lambda^{\prime}}+\frac{C t(1+t)}{(\lambda)_{2}\left(\lambda^{\prime}\right)_{2}}\right\},
\end{gathered}
$$

where

$$
B \equiv \sigma+\frac{a b}{f}, \quad C \equiv(\sigma)_{2}+\frac{2 a b \sigma}{f}+\frac{(a)_{2}(b)_{2}}{(f)_{2}}
$$

Then, from $(2.1)$,

$$
{ }_{4} F_{3}\left(\begin{array}{c|c}
-n, a, b, \quad f+2 \\
c, 3+a+b-c-n, \quad f
\end{array} \mid 1\right)=\frac{(c-a-2)_{n}(c-b-2)_{n}}{(c)_{n}(c-a-b-2)_{n}} \frac{\left(\eta_{1}+1\right)_{n}}{\left(\eta_{1}\right)_{n}} \frac{\left(\eta_{2}+1\right)_{n}}{\left(\eta_{2}\right)_{n}} .
$$

For example, if $a=\frac{3}{2}, b=2, c=\frac{5}{4}$ and $f=1$ we find

$$
Q_{2}(t)=\frac{3465}{256}\left\{1+\frac{40}{99} t-\frac{16}{495} t(1+t)\right\},
$$

which has the zeros $\eta_{1}=-\frac{9}{4}$ and $\eta_{2}=\frac{55}{4}$.

Finally, we consider the case $r=2$ with $m_{1}=m_{2}=1$ (so that $m=2$ ). If we take $a=\frac{1}{3}$, $b=\frac{1}{2}, c=1, f_{1}=\frac{1}{4}$ and $f_{2}=2$, we find the parametric polynomial

$$
Q_{2}(t)=\frac{1}{72}\left\{12+\frac{31}{2} t+\frac{15}{2} t(1+t)\right\}
$$

which has the zeros $\eta_{1}=-\frac{2}{3}$ and $\eta_{2}=-\frac{12}{5}$. Then from (2.1) we obtain

$$
{ }_{5} F_{4}\left(\begin{array}{c|c}
-n, a, b, \frac{5}{4}, 3 \\
c, 3+a+b-c-n, \frac{1}{4}, 2
\end{array} \mid 1\right)=\frac{(c-a-2)_{n}(c-b-2)_{n}}{(c)_{n}(c-a-b-2)_{n}} \frac{\left(\frac{1}{3}\right)_{n}\left(-\frac{7}{5}\right)_{n}}{\left(-\frac{2}{3}\right)_{n}\left(-\frac{12}{5}\right)_{n}} .
$$

Similarly, other results can also be obtained.

Remark. Two other quadratic transformations, different from that in (1.4), have been presented by Miller and Paris $[9$, Section 6$]$ in the form

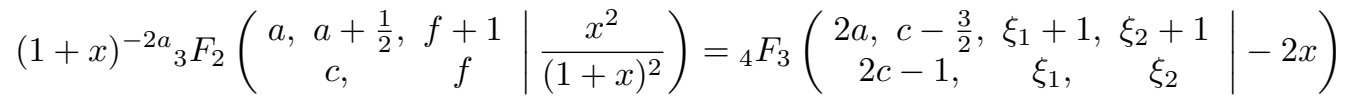

provided $c \neq \frac{3}{2}$, where

$$
\xi_{1,2}=2 f-\frac{1}{2} \pm\left[\left(2 f-\frac{1}{2}\right)^{2}-4\left(c-\frac{3}{2}\right) f\right]^{1 / 2},
$$

and

$$
(1+x)^{-2 a}{ }_{3} F_{2}\left(\begin{array}{cc|c}
a, a+\frac{1}{2}, & f+1 \\
c, & f & \frac{4 x}{(1+x)^{2}}
\end{array}\right)={ }_{4} F_{3}\left(\begin{array}{ccc}
2 a, 2 a-c+1, & \eta_{1}+1, & \eta_{2}+1 \\
c, & \eta_{1}, & \eta_{2}
\end{array}\right)
$$


provided $c \neq 2 a+1$, where

$$
\eta_{1,2}=a \pm\left[a^{2}-(2 a-c+1) f\right]^{1 / 2} .
$$

In the same paper, the authors also derived the extension of both these quadratic transformations in the more general case of $r$ pairs of numeratorial and denominatorial parameters differing by positive integers. It should be pointed out that the results in (3.3) and (3.4) were also obtained in [11] and [12], repectively, following different methods.

Acknowledgement: Y. S. Kim acknowledges the support of the Wonkwang University Research Fund (2012).

\section{References}

[1] Y. S. Kim, M. A. Rakha and A. K. Rathie, Extensions of certain classical summation theorems for the series ${ }_{2} F_{1},{ }_{3} F_{2}$ and ${ }_{4} F_{3}$ with applications in Ramanujan summations, Int. J. Math. Math. Sci. 309503, 26 pages (2010).

[2] Y. S. Kim, A. K. Rathie and R. B. Paris, On a new summation formula for a terminating ${ }_{4} F_{3}(1)$ series with an application, (2012), Preprint.

[3] J. L. Lavoie, F. Grondin, and A. K. Rathie, Generalizations of Watson's theorem on the sum of a ${ }_{3} F_{2}$, Indian J. Math. 34 (1992) 23-32.

[4] J. L. Lavoie, F. Grondin, and A. K. Rathie, Generalizations of Dixon's theorem on the sum of a ${ }_{3} F_{2}$, Math. Comp. 62 (1994) 267-276.

[5] J. L. Lavoie, F. Grondin, and A. K. Rathie, Generalizations of Whipple's theorem on the sum of a ${ }_{3} F_{2}, \mathrm{~J}$. Comput. Appl. Math. 72 (1996) 293-300.

[6] R. S. Maier, $P$-symbols, Heun identities, and ${ }_{3} F_{2}$ identities, Contempory Mathematics 471 (2008) 139-159.

[7] A. R. Miller, A summation formula for Clausen's series ${ }_{3} F_{2}(1)$ with an application to Goursat's function ${ }_{2} F_{2}(x)$, J. Phys. A: Math. Gen. 38 (2005) 3541-3545.

[8] A. R. Miller and R. B. Paris, Euler-type transformations for the generalized hypergeometric function ${ }_{r+2} F_{r+1}(x)$, Zeit. angew. Math. Phys. 62 (2011) 31-45.

[9] A. R. Miller and R. B. Paris, Transformation formulas for the generalized hypergeometric function with integral parameter differences, Rocky Mountain J. Math (2012) [to appear].

[10] M. A. Rakha and A. K. Rathie, Generalizations of classical summation theorems for the series ${ }_{2} F_{1}$ and ${ }_{3} F_{2}$, Integral Transforms Spec. Func. 22 (2011), 823-840.

[11] M. A. Rakha, N. Rathie and P. Chopra, On an extension of a quadratic transformation formula due to Kummer, Math. Commun. 14 (2009), 207-209.

[12] M. A. Rakha, A. K. Rathie and P. Chopra, On an extension of a quadratic transformation formula due to Gauss, International J. Math. Modelling Comp. 1(3) (2011), 171-174.

[13] M. A. Rakha and A. K. Rathie, Extensions of Euler type II transformation and Saalschütz's theorem, Bull. Korean Math. Soc. 48(1) (2011) 151-156.

[14] L. J. Slater, Generalized Hypergeometric Functions, Cambridge University Press, Cambridge, 1966.

[15] R. Vidunas, A generalization of Kummer's identity, Rocky Mountain J. Math. 32(2) (2002) 919-936. 